\title{
Research on the platform of combined multi-functional bioreactor loading performance
}

\author{
Tan Qinsong, Zhang chunqiu, Gao Lilan, Men Yutao \\ Tianjin Key Laboratory of the Design and Intelligent Control of the Advanced Mechatronical System, School of Mechanical Engineering, \\ Tianjin University of Technology, Tianjin 300384, China
}

\begin{abstract}
Mechanical environment plays an important role in the growth of cells, tissues and organs, in the study of mechanobiology and tissue engineering, and loading bioreactor acts as the basic platform. We developed a combined multi-functional bioreactor based on linear motor, and studied on the loading performance of different modules and loading methods. In the rolling / sliding loading module, in the effective distance for $50 \mathrm{~mm}$ case, the bioreactor frequency is $0.01 \mathrm{~Hz}$ to $0.3 \mathrm{~Hz}$. Under tensile and compressive loading module, the minimum amplitude of the bioreactor can reached $0.01 \mathrm{~mm}$ while the frequency can reach $10 \mathrm{~Hz}$. Under the impact test module, in the effective distance for $50 \mathrm{~mm}$ case, acceleration $\mathrm{a}=54.16 \mathrm{~m} / \mathrm{s} 2$ canbe realized. In the high acceleration cyclic loading module, acceleration $8 \mathrm{Gcan}$ be realized. The combined multi-functional bioreactor provides conditions for the study related mechanobiology and tissue engineering.
\end{abstract}

\section{Introduction}

At present, there is a big challenge to repair the defect of human body tissue. Tissue engineering is an ideal method for repairing the tissue defect. Tissue engineering need to construct artificial tissue in vitro, and bioreactor plays a crucial role in tissue engineering, They can be constructed on the cell scaffold in a controllable mechanical environment, even large-scale industrial production [1].

Bioreactor is one of the key technologies in tissue engineering. the ability of accurately simulated tissue, organ and cell mechanics environment is the focus of attention, and each tissue type (such as skin, bone, blood vessel, and cartilage) may require designing the different bioreactor [2]. Therefore the design of special tissue bioreactor should be based on comprehensive understanding of the biological and engineering problems, and typical engineering problems such as reliability, reproducibility, scalability, and security should be considered [3,4], a lot of bioreactor systems have been developed and are expected to be high [4,5]. Cartilage tissue engineering bioreactor mainly types includes mechanical stirring bioreactor, perfusion - hydraulic type bioreactor, rotating wall bioreactor, air lift bioreactor, micro load type bioreactor and other bioreactor. Although there are various bioreactors in colleges, universities and research institutes, but linear motor driven bioreactor is relatively rare at home and abroad. In addition, compared to ball screw linear motor has the advantages of simple structure, high precision, high speed and high acceleration etc.
This paper conducted a deep study of the combined multi-functional bioreactor based on linear motor. Provided references for mechanical biology and tissue engineering experiment, and determined the suitable frequency, amplitude and maximum acceleration of the bioreactor. According to the main modules of the loading platform, study on the loading performance from the following aspects.

\section{Instruments}

Combined multi-functional bioreactor not only can realized rolling / sliding and combine rolling and sliding loading, but also carry on high frequency / low frequency and double frequency loading, see Fig. 1.

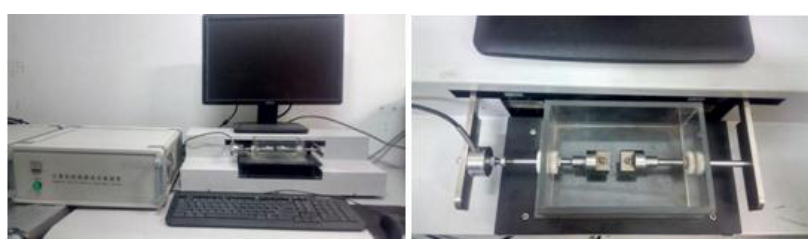

Figure 1. Combined multi-functional bioreactor.

The combined multi-functional bioreactor is divided into two parts: the hardware system and the software system. The hardware system includes the linear motors, drivers, sensors, PMAC motion control card, clamp, base and hood. The software system includes man-machine interface program and motion program in PMAC motion control card (Fig. 2). 
The parameters of main components: Effective distance of the linear motor $50 \mathrm{~mm}$, continuous force is $38 \mathrm{~N}$, peak force is $111 \mathrm{~N}$. Resolution of grating ruler is 5 microns.

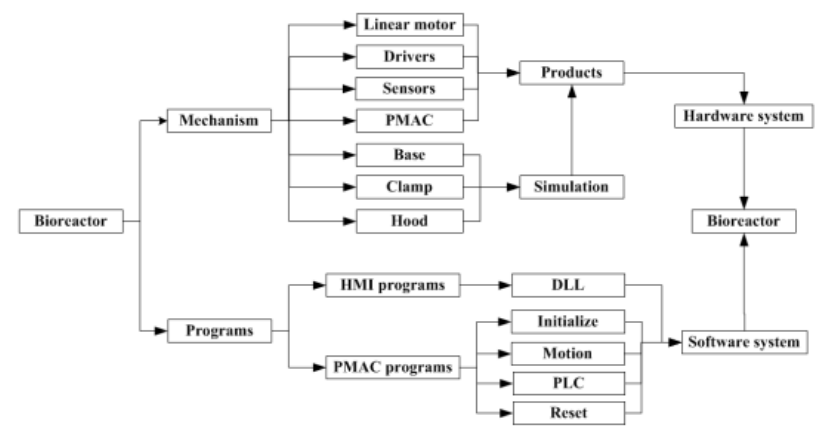

Figure 2. System diagram.

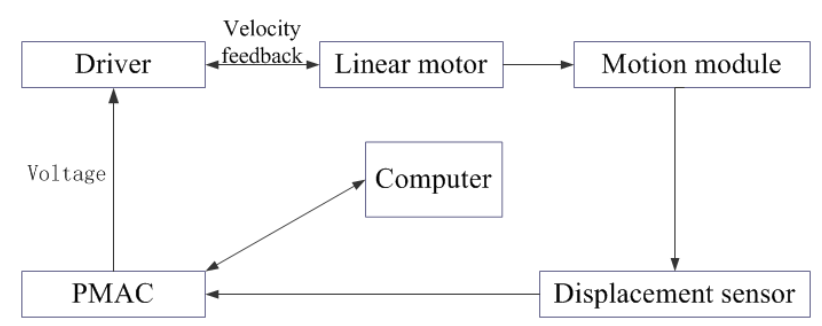

Figure 3. Control principle diagram.

The combined multi-functional bioreactor control process: Firstly, the computer send commands to the PMAC motion control card. Secondly, the result of compared input of computer and feedback of position sensor as the input of drivers. Finally, compare the input of drivers with feedback of speed sensor and the results of compared drive motor to move so that achieve the intended function in the experiment.

\section{Methods}

\subsection{Study on the tension and compression loading module}

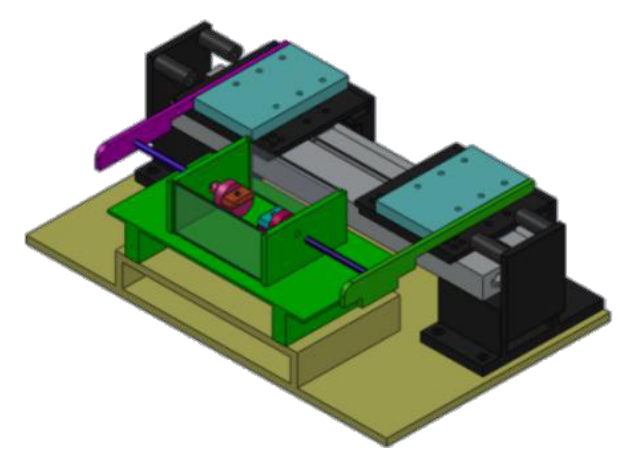

Figure 4. The tension and compression loading module

Theory analysis: the linear motor motion model is divided into triangular, trapezoidal and $\mathrm{S}$ type curve. S type curve motion model of linear motor be influenced by variable acceleration. It is bad for loading experiment, and the linear motor life would also be affected. Trapezoid motion curve because of the uniform motion stage, has a protective effect for the motor, but the frequency is not too high. In addition, for short stroke, linear motor is recommended to use triangular velocity mode (no uniform). The trapezoidal velocity model will be more efficient for long stroke. So we use triangular of linear motor mode for theoretical and experimental analysis (Fig. 5).

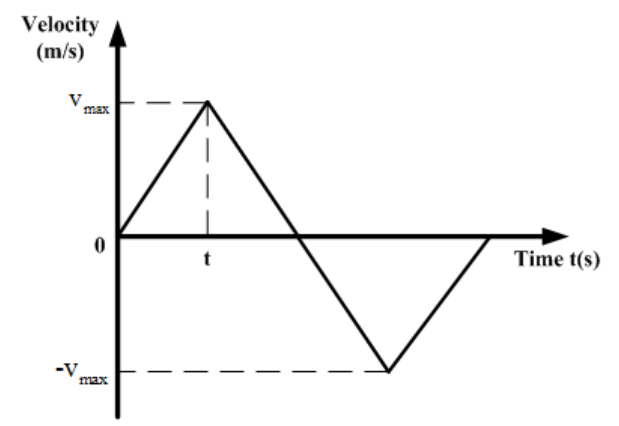

Figure 5. The triangle mode speed-time.

$$
f=\frac{1}{T}=\frac{1}{4} \sqrt{\frac{F}{2 S m}}=\frac{1}{4} \sqrt{\frac{2 F}{m L}}, F \text { is the force of the }
$$

linear motor. $L$ is the distance of the linear motor. $m=0.925 \mathrm{~kg}$ is the quality of mover. So we can know the frequency $f=10.134 \mathrm{~Hz}$, the max frequency $f=17.32 \mathrm{~Hz}$.

The experimental results are as follows:

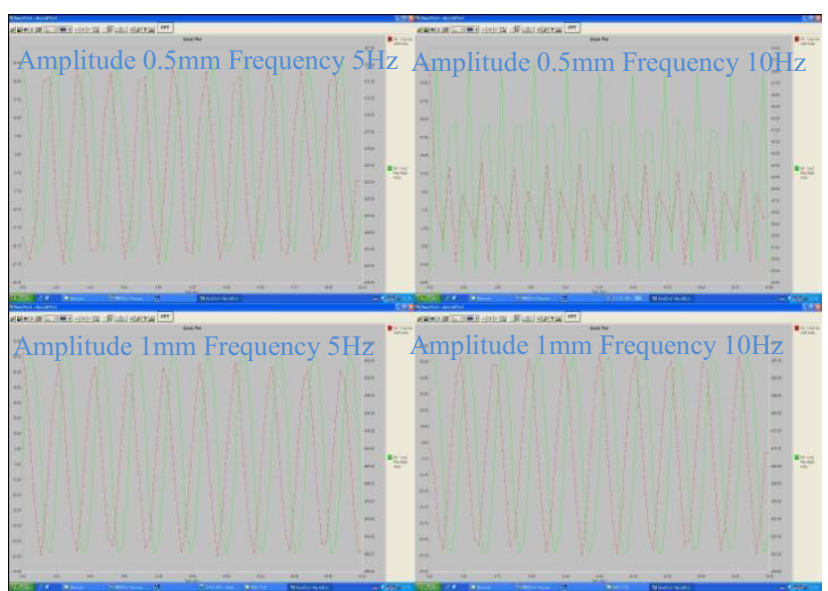

Figure 6. The motion curves.

Note: the green curve stand for displacement-time. the red curve is speed-time.

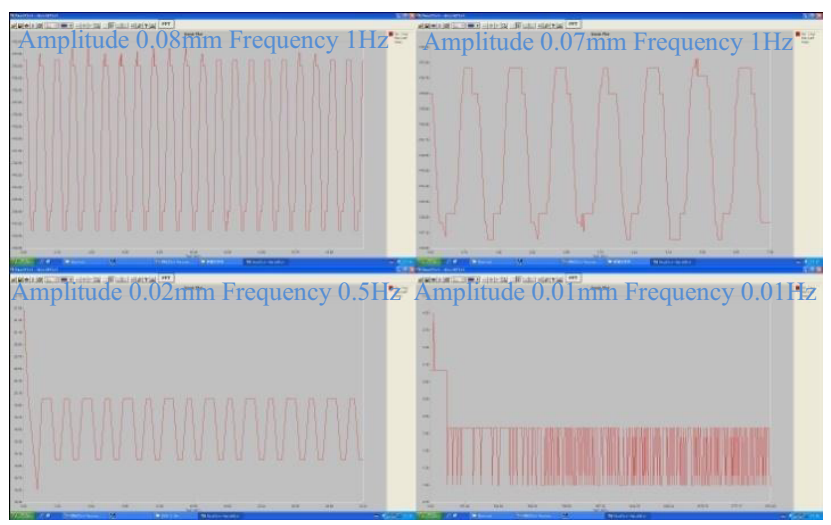

Figure 7. The motion curves

Note: the red is displacement-time curve. 
Analysis:experimental results are basically consistent t o theoretical analysis results. The experiments (Fig. 6) show that the frequency of the bioreactor can reach $10 \mathrm{~Hz}$. With the increase of the frequency or the decrease of the displacement, the motion characteristics of the system will be unstable. The frequency of the system is inversely proportional to the square root of the displacement. A large number of experimental data statistics show that, the system has better movement characteristics when the amplitude are $80 \mu \mathrm{m}, 20 \mu \mathrm{m}, 10 \mu \mathrm{m}$ and the frequency are $1 \mathrm{~Hz}, 0.5 \mathrm{~Hz}, 0.01 \mathrm{~Hz}$ (Fig. 7).

\subsection{Research on the rolling and sliding pressure module}

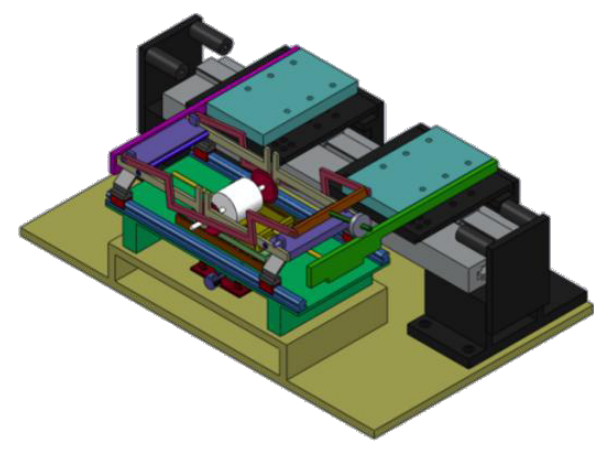

Figure 8. The Rolling and sliding pressure module.

In rolling and sliding loading test, the roller has larger displacement than tension and compression loading, in addition the effective stroke of the linear motor is $50 \mathrm{~mm}$, so the given amplitude is $50 \mathrm{~mm}$ and $30 \mathrm{~mm}$, the experimental results are as below:

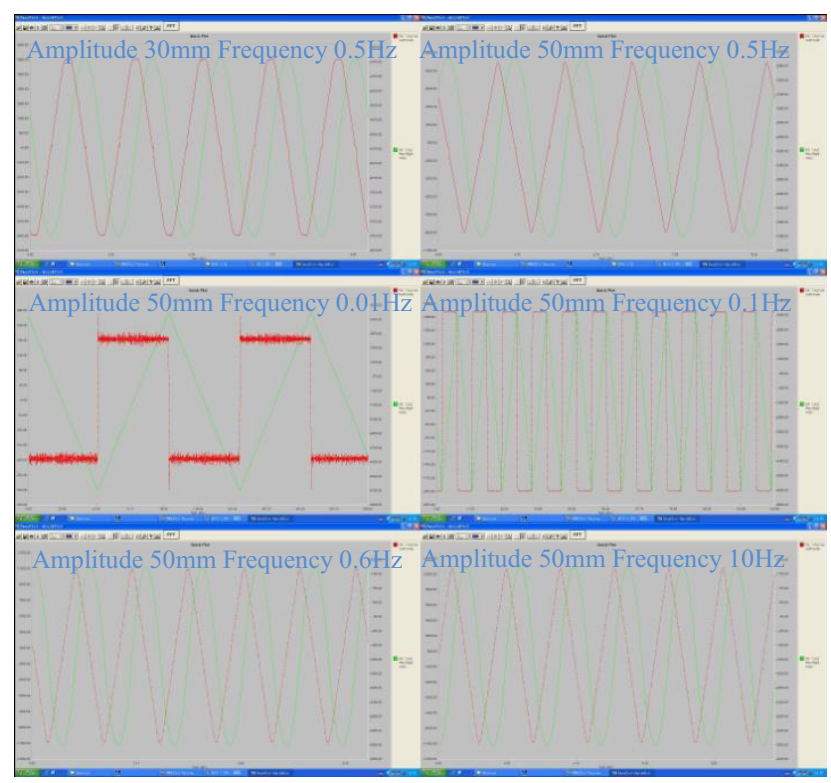

Figure 9. The motion curves.

Note: the green curve stand for displacement-time. the red curve is speed-time.

Analysis: In the same frequency, the larger the amplitude, the better the performance of the bioreactor. In the effective stroke $50 \mathrm{~mm}$, the system has the accurate amplitude and frequency when the given frequency is
$0.01 \mathrm{~Hz}$ to $0.3 \mathrm{~Hz}$. The frequency of $0.3 \mathrm{~Hz}$ to $0.6 \mathrm{~Hz}$, the system has accurate frequency, but the amplitude has great error. When the frequency is larger than $0.6 \mathrm{~Hz}$, the actual amplitude and the actual frequency have large errors (Fig. 9).

\subsection{Research on the high acceleration of cyclic loading experiment}

Theory analysis: the selection of linear motor includes calculating the maximum force and continuous force. The maximum force is determined by the mass of the moving load and the maximum acceleration. Force $=$ total mass * acceleration + friction + external stress, because of the small friction of linear motor, the experiment is carried out under no load, so:

$$
F=m a=0.925 a=111 N \quad a=120 \mathrm{~m} / \mathrm{s}^{2}=12 \mathrm{G}
$$

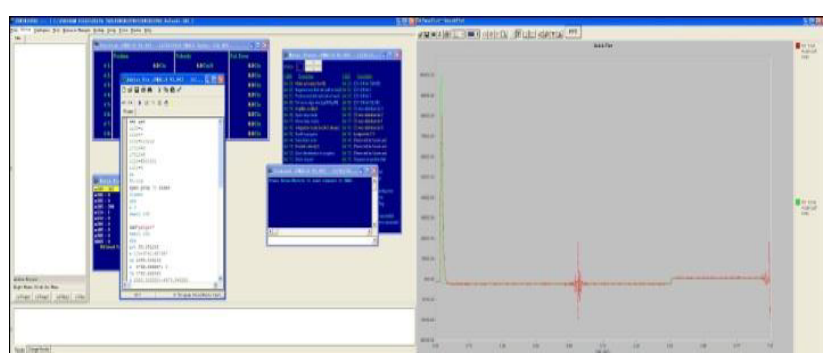

Figure 10. The programming interface and acceleration-time curves

Note: the green curve stand for theoretical acceleration-time. the red curve is actual acceleration-time.

The experimental results show that the theoretical acceleration of PMAC program design is $10 G$ while the actual acceleration of the motor can reach about $8 G$, and the actual motion curve and the theoretical curve change rules are in agreement.

\subsection{Study on the impact test}

Giving amplitude and frequency of linear motor, and simulating impact test of linear motor. Test results are as follow:

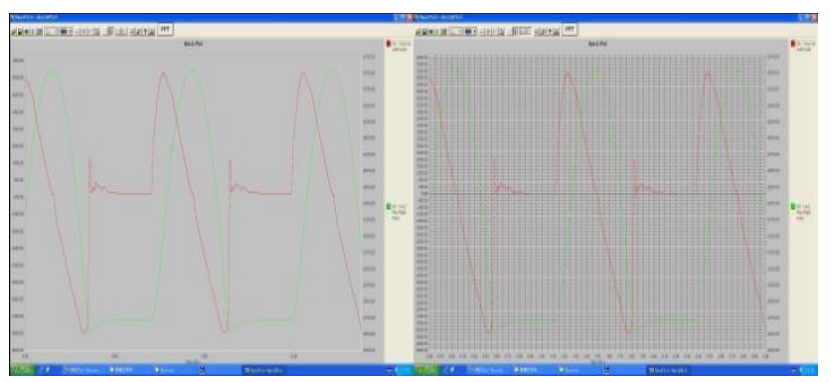

Figure 11. The motion curves.

Note: the green curve stand for speed-time. the red curve is displacement-time.

Experimental results: through analysis curve, we can know impact acceleration and impact force of the experimental platform are as follows: 


$$
a=54.16 m / s^{2} F=m a=0.925 \times 54.16=50.1 N .
$$

\section{Conclusion}

1) In tension / compression loading module, experiments results show that: With increasing frequency, the motion characteristics of the system is more unstable. The minimum amplitude of the combined multi-function bioreactor loading platform can reach $0.01 \mathrm{~mm}$, the frequency range is $0.01 \mathrm{~Hz}$ to $10 \mathrm{~Hz}$. Theoretical analysis shows that the frequency of the system is inversely proportional to the square root of the displacement.

2) Under the rolling and sliding loading module, experimental results show that: The loading amplitude of the experimental platform can reach $50 \mathrm{~mm}$. The frequency range from $0.01 \mathrm{~Hz}$ to $0.3 \mathrm{~Hz}$ can guarantee the accuracy of the actual amplitude and the actual frequency.

3) Under cyclic loading module, maximum acceleration of the experimental platform can reach $8 \mathrm{G}$. Actual motion curves and the theory of motion curve is roughly consistent, indicating that programs and control of the experimental platform are right. If you want to achieve greater acceleration, you can choose a larger force or longer distance of linear motor.

4) Under the impact test module, impact acceleration and impact force of the experimental platform are as follows:

$$
a=54.16 \mathrm{~m} / \mathrm{s}^{2} F=m a=0.925 \times 54.16=50.1 \mathrm{~N} .
$$

Because the linear motor has many advantages such as high acceleration, high precision, simple structure and so on. So the linear motor can be used for the development of high frequency, great acceleration and impact of bioreactor, will also be more and more applied to the development of other kinds of bioreactor.

\section{Acknowledgements}

The project was partly supported by the National Natural Science Key Foundation of China (Nos.11432016) and the National Natural Science Foundation of China (Nos. $11372221,11572222,11402171)$.

\section{References}

1. T. Takebe, S. Kobayashi, H. Kan, H. Suzuki, Y. Yabuki, M. Mizuno, T. Adegawa, T. Yoshioka, J. Tanaka, J. Maegawa, H. Taniguchi, Human elastic cartilage engineering from cartilage progenitor cells using rotating wall vessel bioreactor, Transplant. Proc. 44 (2012) 1158-1161.

2. Ratcliffe, A. and Niklason, L. E.: Bioreactors and bioprocessing for tissue engineering. Ann. N.Y. Acad. Sci., 961, 210-215 (2002).

3. Martin, I., Wendt, D., and Heberer, M.: The role of bioreactors in tissue engineering. Trends Biotechnol., 22, 80-86(2004).

4. Naughton, G. K.: From lab bench to market: critical issues in tissue engineering. Ann. N.Y. Acad. Sci., 961, 372-385(2002).

5. Griffith, L. G. and Naughton, G.: Tissue engineering - current challenges and expanding opportunities. Science, 295, 1009-1014 (2002). 